\title{
Erratum to: Preoperative Predictive Factors of Successful Weight Loss and Glycaemic Control 1 Year After Gastric Bypass for Morbid Obesity
}

\author{
Silvia Palmisano ${ }^{1} \cdot$ Marta Silvestri $^{1} \cdot$ Michela Giuricin $^{1} \cdot$ Edoardo Baldini $^{2}$. \\ Simone Albertario $^{2}$ - Patrizio Capelli ${ }^{2}$ - Bernardo Marzano ${ }^{3}$ - Giovanni Fanti ${ }^{3}$. \\ Aron Zompicchiatti ${ }^{3}$ Paolo Millo ${ }^{4}$. Massimiliano Fabozzii ${ }^{4}$. \\ Riccardo Brachet Contul ${ }^{4} \cdot$ Elisa Ponte $^{4} \cdot$ Rosaldo Allieta $^{4} \cdot$ Nicolò de Manzini $^{1}$
}

Published online: 1 March 2016

(C) Springer Science+Business Media New York 2016

Erratum to: OBES SURG (2015) 25:2040-2046

DOI 10.1007/s11695-015-1662-2

Due to a coding error an author's name was presented incorrectly in the XML (online) version of this article - the name should be presented as Brachet Contul, R.

The online version of the original article can be found at http://dx.doi.org/ $10.1007 / \mathrm{s} 11695-015-1662-2$.

Silvia Palmisano

spalmisano@units.it

Marta Silvestri

silvestri_marta@libero.it

Michela Giuricin

michelagiuricin@libero.it

Edoardo Baldini

e.baldini@ausl.pc.it

Simone Albertario

s.albertario@ausl.pc.it

Patrizio Capelli

p.capelli@ausl.pc.it

Bernardo Marzano

bernardo.marzano@gmail.com

Giovanni Fanti

giovanni.fantipn@gmail.com

Aron Zompicchiatti

aronillo@yahoo.it

Paolo Millo

pmillo@ausl.vda.it
Massimiliano Fabozzi

mfabozzi@ausl.vda.it

Riccardo Brachet Contul

rbrachetcontul@ausl.vda.it

Elisa Ponte

eponte@ausl.vda.it

Rosaldo Allieta

rallieta@ausl.vda.it

Nicolò de Manzini

demanzini@units.it

Department of Medical, Surgical and Health Sciences, General Surgery Clinic, University Hospital of Trieste, Strada di Fiume, 447, Trieste, Italy

2 Unità Operativa di Chirurgia Generale, Toracica e Vascolare, Ospedale "Guglielmo da Saliceto", via Taverna 49, 29122 Piacenza, Italy

3 Department of Surgery, Santa Maria degli Angeli Hospital, Via Montereale 24, 33170 Pordenone, Italy

4 Department of General Surgery, Bariatric and Metabolic Unit, "Umberto Parini" Regional Hospital of Aosta, Viale Ginevra 3, Aosta, Italy 